\title{
Comparative analysis of explanted DBS electrodes
}

\author{
Michele Rizzi $^{1,2}$ - Alessandro De Benedictis ${ }^{3} \cdot$ Giuseppe Messina $^{1} \cdot$ Roberto Cordella $^{1}$. \\ Davide Marchesi $^{4} \cdot$ Raffaella Messina $^{3} \cdot$ Federica Penner $^{1}$ • Angelo Franzini ${ }^{1}$. \\ Carlo Efisio Marras ${ }^{3}$
}

Received: 17 June 2015 / Accepted: 27 August 2015 /Published online: 7 September 2015

(C) Springer-Verlag Wien 2015

\begin{abstract}
Background Hardware-related complications frequently occur in deep brain stimulation. Microscopy and spectroscopy techniques are effective methods for characterizing the morphological and chemical basis of malfunctioning DBS electrodes. A previous report by our team revealed the morphological and chemical alterations on a malfunctioning explanted electrode when it was compared to a new device. The aim of this preliminary study was to verify whether these morphological and chemical alterations in the materials were a direct result of the hardware malfunctioning or if the failure was correlated to a degradation process over time.

Methods Two DBS electrodes were removed from two patients for reasons other than DBS system impairment and were analyzed by a scanning electron microscope and by an energydispersive X-ray spectroscopy. The results were compared to a malfunctioning device and to a new device, previously analyzed by our group.

Results The analysis revealed that the wear of the polyurethane external part of all the electrodes was directly correlated with the duration of implantation period. Moreover, these alterations were independent from the electrodes functioning and from parameters used during therapy.
\end{abstract}

Michele Rizzi

michele.rizzi@live.it

1 Department of Neurosurgery, IRCCS Foundation Neurological Institute "Carlo Besta", Milan, Italy

2 School of Neurosurgery, University of Milan, Milan, Italy

3 Department of Neuroscience and Neurorehabilitation Division of Neurosurgery, Bambino Gesù Children's Hospital, IRCCS, Rome, Italy

4 Filarete Foundation, Milan, Italy
Conclusions This is the first study done that demonstrates a time-related degradation in the external layer of DBS electrodes. The analyses of morphological and chemical properties of the implanted devices are relevant for predicting the possibility of hardware's impairment as well as to improve the bio-stability of DBS systems.

Keywords Scanning electron microscope $\cdot$ Energy dispersive $\mathrm{X}$-ray · Environmental biodegradation · Electrodes analysis . Explanted electrodes $\cdot$ DBS

\section{Introduction}

Deep brain stimulation (DBS) is an effective treatment for different neurological and psycho-affective disorders $[5,15]$. Despite progressive technological advancement, hardwarerelated complications, including lead fracture or migration and infections, have been observed $[1,12]$.

Currently, high-magnification microscopy (scanning electron microscopy, SEM) and chemical characterization through spectroscopic techniques (energy-dispersive X-ray spectroscopy, EDS) are extensively used to perform failure analysis tasks in many medical fields $[11,16]$, particularly in the case of defects arising from material failures. These methods could be implemented in the study of explanted DBS electrodes in order to analyze the structural basis of the impairment of implanted systems.

In a previous report by our team [7], we revealed the morphological and chemical alterations on a malfunctioning explanted electrode in comparison with a new device, which was adopted as a control. Since a correlation between the observed changes and the electrode's breakdown was not clearly demonstrated, a time-related influence was considered to be a cause of the device malfunction. 
Our report shows a preliminary comparative analysis between a malfunctioning explanted electrode, two electrodes that were removed from two patients for reasons other than implanted system impairment and a new electrode.

\section{Materials and methods}

\section{Patients}

\section{Patient 1}

Patient 1 is a 25 years-old male with an onset of walking dystonia at the age of six. The patient was diagnosed with idiopathic primary generalized dystonia at 11 years-old. DBS of the globus pallidus internus (GPi) was performed at the age of 16 , in 2005. Consequently to stimulation, the patient remarkably improved and he was walking independently 1 year later (severity score at BFMDRS: 66). At the age of 19 , a worsening of dystonia involving the lower limbs and the right upper limb appeared (BFMDRS: 71); the electrode's impedance was higher than $2000 \mathrm{Ohm}$. In June 2010, the patient agreed to have the faulty electrodes removed and replaced with a new lead (3389; Medtronic) on the left side. The procedure was successful and without complications. During the last follow-up (January 2014), DBS resulted to be effective in the treatment of dystonic symptoms (BFMDRS: 60) (Table 1).

\section{Patient 2}

This patient is a 30 -year-old female who began experiencing seizures in 2003. Cortical and subcortical biopsies of the left frontal lobe suggested that Rasmussen encephalitis was the etiology. Four years after the onset, the patient developed a drug-resistant epilepsia partialis continua (EPC). Given that cognitive, motor, and language functions were good, a left caudal zona incerta (cZI) DBS was proposed in October 2007 (11) and significant improvements in the frequency of seizures was reported. In 2009, the patient had an EPC refractory to the stimulation parameters arrangement to the AED dose augmentation and to the immunomodulatory treatment. The DBS system revealed correct impedance values and did not show any other signs of malfunctioning. Thus, the left motor cortex was surgically removed and the seizures were completely controlled. Moreover, the patient had requested for the DBS system to be switched off because it was ineffective. In March 2013, the entire DBS system was removed (Table 1).

\section{Patient 3}

Patient 3 is a 22-year-old male whose clinical history started at the age of 3 with a progressive spastic paraparesis associated to
Table 1 General characteristics of the patients

\begin{tabular}{|c|c|c|c|c|}
\hline & Diagnosis & $\begin{array}{l}\text { Age at } \\
\text { implantation } \\
\text { (years) }\end{array}$ & $\begin{array}{l}\text { Period of device } \\
\text { implantation } \\
\text { (years) }\end{array}$ & $\begin{array}{l}\text { DBS system removal } \\
\text { motivation }\end{array}$ \\
\hline Pt 1 & Dystonia & 10 & 4 & $\begin{array}{l}\text { Not specified } \\
\text { malfunctioning }\end{array}$ \\
\hline Pt 2 & Epilepsy & 23 & 6 & $\begin{array}{l}\text { Loss of efficacy, } \\
\text { without malfunction }\end{array}$ \\
\hline Pt 3 & Dystonia & 10 & 10 & $\begin{array}{l}\text { DBS system } \\
\text { infection without } \\
\text { malfunctioning }\end{array}$ \\
\hline
\end{tabular}

dystonic movements of the right upper arm. The clinical picture progressively worsened and a generalized dystonia appeared. A diagnosis of primary DYT1- dystonia was posed (BFMDRS, severity score: 79.5; disability: 19). A drug-resistant status distonicus (SD) appeared at the age of 10. Bilateral GPi-DBS was performed in 2002; internal pulse generators (IPGs) were switched on and 2 days later the SD disappeared (BFMDRS, severity score: 63; disability: 10). In 2011, an infection of the connection cable occurred; antibiotic treatment was not effective and removal of the cable became necessary, followed by dystonia worsening. Infection extended to the IPGs and successively to the extracranial portion of the lead. The patient underwent the removal of the entire left DBS system in 2012 and a few months later a left pallidotomy was performed and resulted in improvement of the disease (Table 1).

\section{Electrode analysis}

All of the electrodes were carefully removed from the patients, without any complications or damage to the hardware. The electrodes were preserved in three different surgical specimen boxes after rinsing them with saline solution. The study included both the explanted electrodes (3389; Medtronic, length $25 \mathrm{~cm}$ ) and a new lead produced by the same company (3389; Medtronic, length $40 \mathrm{~cm}$ ). Depending on the facilities availability, the analysis through SEM-EDX techniques was not performed the same day of removal.

Scanning electron microscopy characterization of the electrode had been performed at the Micro and NanoFabrication Platform of Fondazione Filarete, Milan, Italy, using a Zeiss Sigma Field Emission Scanning Electron Microscope (FE-SEM). The chemical composition analysis of the samples was performed using a Bruker Quantax 400 EDS detector $\left(30 \mathrm{~mm}^{2}\right.$ XFlash silicon drift detector) installed on the SEM.

SEM was carried out to describe the morphological features of the electrodes at different magnifications, while EDX was performed to analyze the chemical properties of the materials. 


\section{Results}

The macroscopic inspection of the electrodes removed from patients 2 and 3 did not show any alterations. In a portion of the extracranial electrode of patient 1 , we found browncolored areas beneath the external layer of the electrode (i.e., outer jacket tubing). Moreover, the metallic coils appeared to be distorted and stretched throughout different parts of the electrode. The conductivity of this electrode reported zero (i.e., open circuit), while that of patients two and three was preserved [7].

The morphological analysis was performed through SEM, with different magnification on the outer jacket tubing and on the internal insulating jacket. The former was made of polyurethane ( $80 \mathrm{~A}$ urethane), while the latter was a fluoropolymeric structure, which isolates each single coil of the electrode which is composed by platinum and iridium.

Figure 1 shows the comparison between each electrode at the level of the outer jacket tubing. In patient 1, cracklike forms, perpendicular to the lead axis, extended above the lead surface (282x) (Fig. 1a); in patient 2, star-like forms appeared to be more evident at the level of the jacket near the stimulating contact (347x) (Fig. 1b); in patient 3, crack-like forms, always perpendicular to lead direction, resulted more evident with the disappearance of the classical smooth surface (Fig. 1c). The right upper corner of Fig. 1a, b and c shows a more detailed look at the outer jacket polymer surface, by using a higher magnification. In the same figure, the alterations extended to the whole surface, with a striking loss of the initial characteristics. Nevertheless, no communication between the inner part of the lead and the external lead was observed. As shown in Fig. 2, only a superficial part of the outer jacket tubing was severely deteriorated: the horizontal arrow (letter b) shows the internal border of the outer jacket tubing, while the vertical arrow (letter a) points towards the external surface. The magnification of 14.66 Kilo-X detailed the above described alterations on the outer jacket tubing in patients 2 and 3 (Fig. 3a, b).

The internal insulating jacket was explored by carefully removing the outer jacket tubing. In patient 1 , the electrode jacket was covered by remnants of degenerated material (Fig. 4a) [7]. The internal insulating jacket of patients 2 and 3 was found to be well preserved (Fig. 4b and c).

EDX analysis revealed that the outer jacket tubing of the four electrodes presented a chemical composition compatible with the typical composition of polyurethanes. A tendency of N/C ratio increase with the time to removal was observed (Table 2). The internal insulating jacket revealed that the used electrodes had a prevalence of carbon (mean value of $50 \%$ against $38 \%$ of the control electrode) and a minor percentage of fluorine (mean value $43.3 \%$ against $61 \%$ of the new electrode) (Table 3 ). The chemical structure of the material partially covering the internal insulating jacket in patient 1 was composed of $63 \%$ of carbon, $3.5 \%$ fluorine, $12 \%$ of oxygen, and $11 \%$ nitrogen (Table 3) [7].

\section{Discussion}

Despite the widespread use of DBS and other neuromodulation procedures in clinical practice, clinicians are often faced with different adverse events related to the hardware that is implanted $[1,12]$. In order to investigate the morphological and chemical features of these devices, preclinical studies are required. Relevant information could also be collected by analyzing the DBS systems that are explanted because of complications such as lead fracture, migration or disconnection of the electrode, connection cable, or pulse generator infection. SEM and EDX are effective methods supporting these studies.

Previously, our group reported a comparative evaluation between two electrodes, one explanted for malfunctioning and a new one [7]. We hypothesized that the observed modifications were secondary to the overheating of the lead that is associated with the partial degradation of the internal structure. However, a direct cause for the malfunctioning was not found. In particular, it was not possible to determine if the alterations were truly relevant to the patient's problem or if there were more frequent phenomenon occurring in the majority of the DBS electrodes. For these reasons, we described two leads that were removed from two different patients for reasons other than malfunctioning. This is, to the best of our knowledge, the first study in which a similar analysis has been performed, allowing us to speculate about the effects of wear over time.

In all of the explanted leads, both the SEM morphological analysis and the EDX chemical study revealed alterations of the outer jacket tubing directly correlated with the duration of implantation period (Tables 1, 2 and Figs. 1, 2, 3). The most relevant alterations resulted in patient 3 , where the electrodes were implanted for a period of 10 years (Table 1). These considerations support the hypothesis that the polyurethane (PU) wears over time. The outer jacket tubing is composed of a PU, notably the $80 \mathrm{~A}$ Urethane. Studies focusing on cerebral implanted materials have never been performed, but previous studies on PUs implanted for other medical reasons, such as cardiac valves or breast implants, described the problem of long-term bio-stability of these materials $[2,9,10,14]$. A complete review revealed the "micro-fissure phenomenon", also known as environmental stress cracking (ESC) or environmental biodegradation (EB). Different aspects appear to contribute to EB: residual polymer surface stress, introduced 

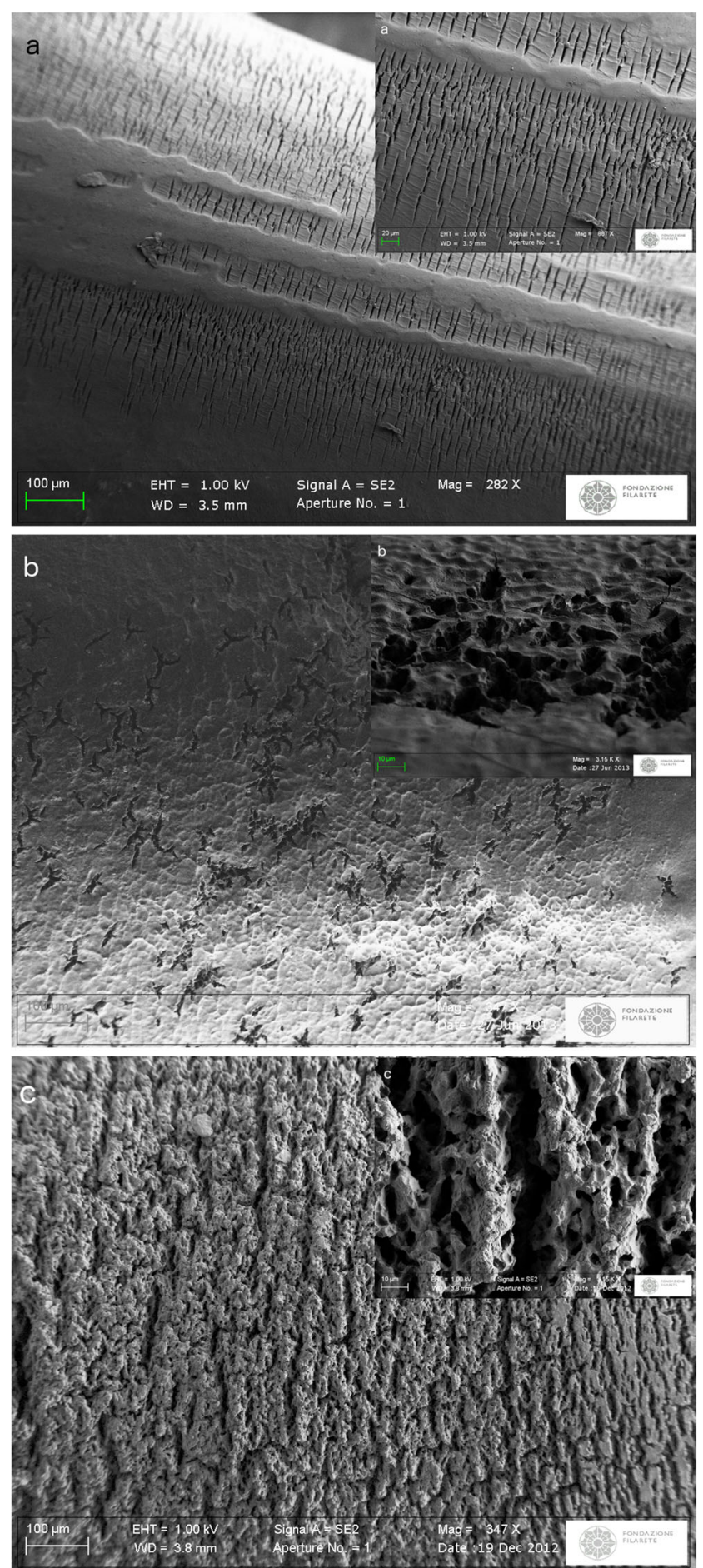

Fig. 1 Morphology of the DBS leads analyzed by SEM: imaging of the outer jacket tubing at similar magnification: a patient 1's DBS lead, showing crack-like alterations, at $\times 282$ magnification. In the right upper corner, a particular at $\times 867$ magnification (Marras et al. 2011); b patient 2 's DBS lead, showing star-like alterations, at $\times 347$ magnification. In the right upper corner, a particular at $\times 3150$ magnification; d patient 3 's DBS lead, showing crack-like alterations with the disappearance of the classical smooth surface, at $\times 347$ magnification. In the right upper corner, a particular at $\times 3150$ magnification

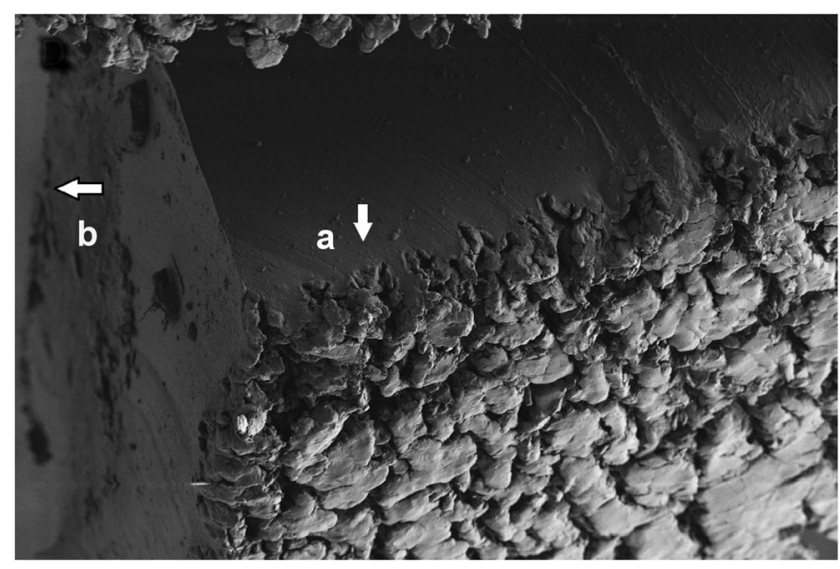

Fig. 2 Patient 3. Section of the outer jacket tubing: particular through SEM analysis at $\times 1010$ magnification. The vertical white arrow and the letter " $a$ " indicates the external part of the outer jacket tubing (close to the degenerated surface); the horizontal white arrow and the letter " $b$ " indicates the internal part

during fabrication, hydrolytic degradation, and bulk oxidation catalyzed by corrosion of the metallic component. In our
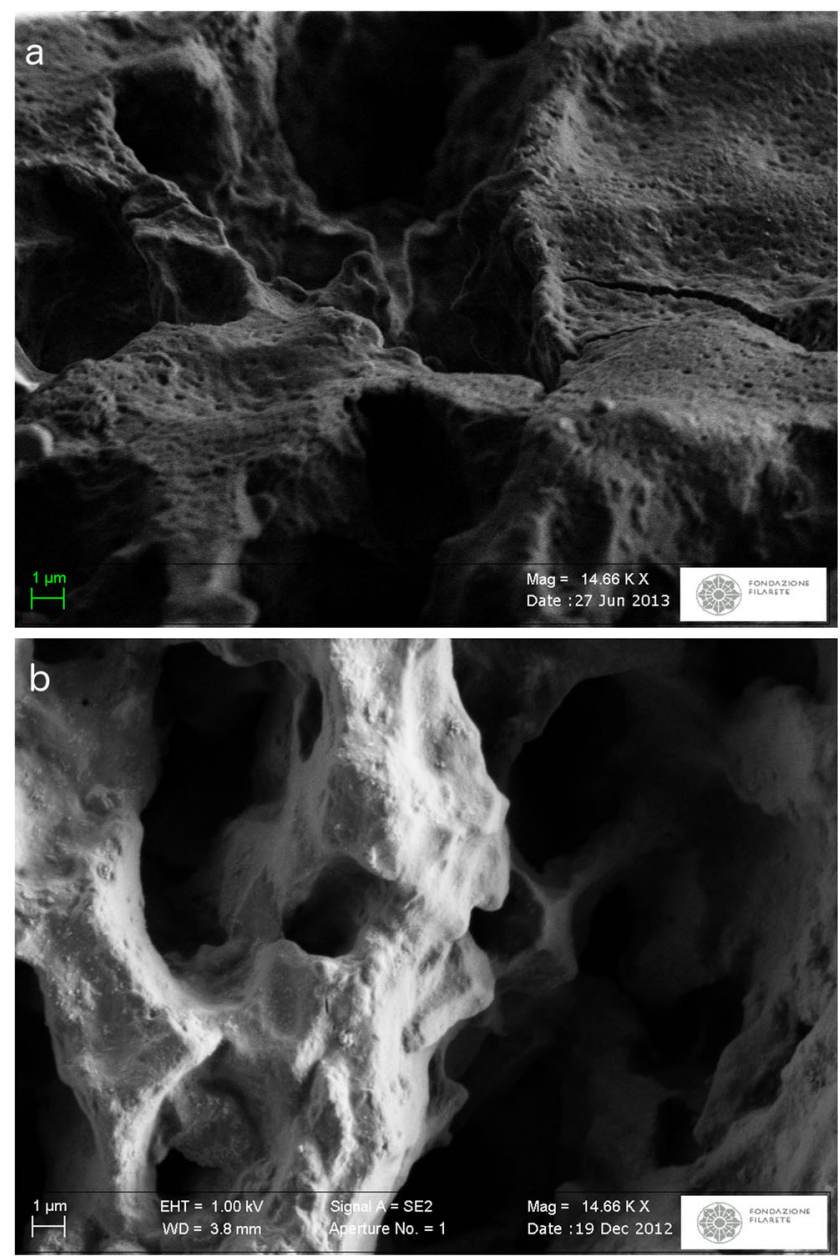

Fig. 3 Morphology of the DBS leads analyzed by SEM: imaging of the outer jacket tubing fissurations at $\times 14660$ magnification: a patient 2 's DBS lead; b patient 3's DBS lead 

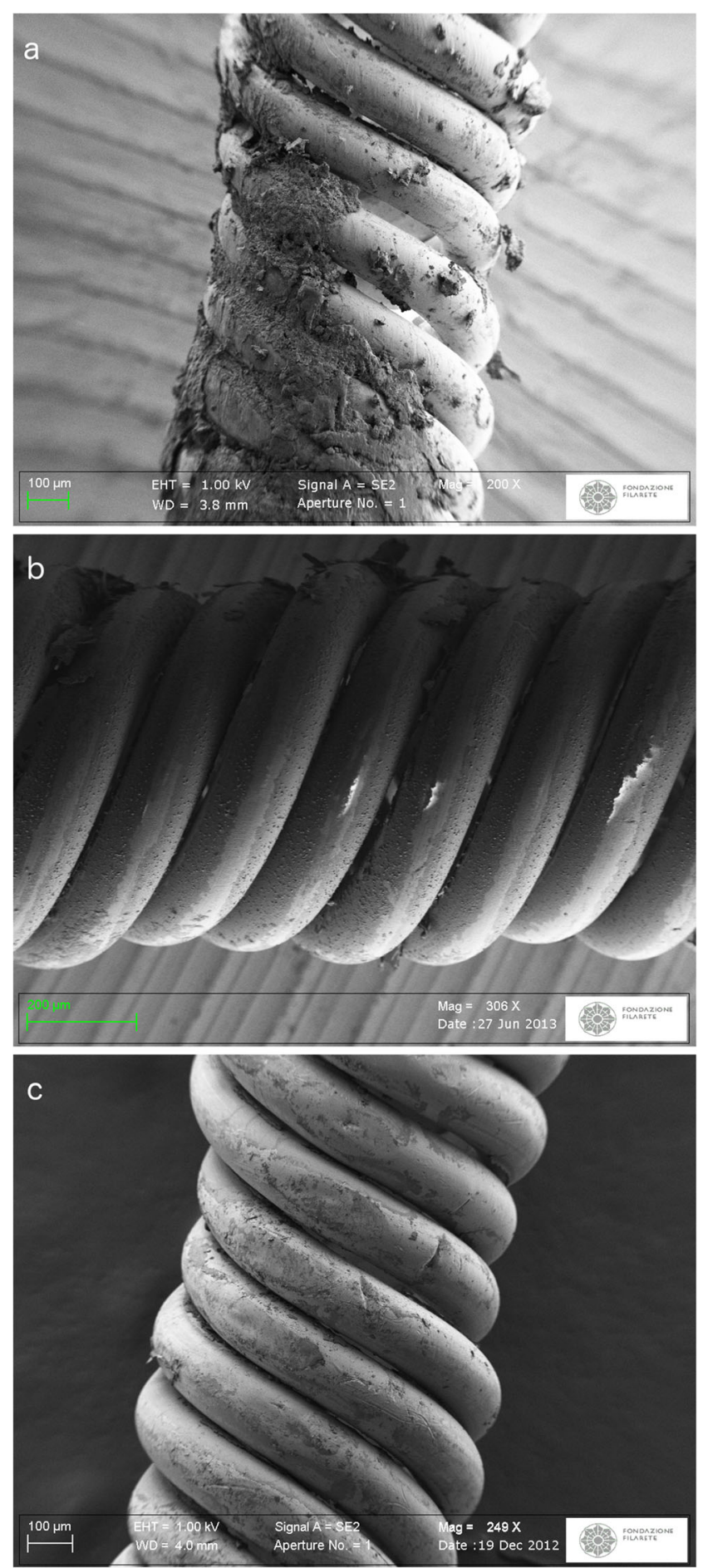

Fig. 4 Morphology of the DBS leads analyzed by SEM: imaging of internal insulating jacket at similar magnifications: a patient 1's DBS lead $(\times 200)$ (Marras et al. 2013); b patient 2's DBS lead $(\times 306)$; c patient 3's DBS lead $(\times 249)$

study, the oxidation process probably did not have a prominent role in the outer jacket tubing degradation: platinumiridium wires were insulated by the internal insulating jacket and the internal layer of the outer jacket tubing was not affected by the EB. Hydrolytic degradation $[10,13]$ is carried out
Table 2 Comparison between the chemical composition of the outer jacket tubing of the analyzed electrodes

\begin{tabular}{lccc}
\hline & $\mathrm{C}$ & $\mathrm{O}$ & $\mathrm{N}$ \\
\hline Patient 1 & $84 \%$ & $9 \%$ & $7 \%$ \\
Patient 2 & $77 \%$ & $10 \%$ & $10 \%$ \\
Patient 3 & $75 \%$ & $14 \%$ & $9 \%$ \\
Mean (pt 1, 2,3) & $78.6 \%$ & $11 \%$ & $8.6 \%$ \\
Control electrode & $81 \%$ & $13 \%$ & $6 \%$ \\
Equilibrium & $-2.4 \%$ & $-2 \%$ & $+2.6 \%$ \\
\hline
\end{tabular}

through the immunogenic response of monocyte-mediated macrophages (MDM) and foreign body giant cells (FBGC), inducing enzymatic activation. In the human central nervous system, this role belongs to the microglia. As stated by Moss et al., DBS leads raise a FBGC reaction around them in case of brain implantation [8]. In patient 3, where the lead was implanted for 10 years, EB resulted notable. In this case, multiple and prolonged extracranial DBS system infections caused by Serratia marcescens led to the removal of the electrode. So far, the enzymatic hydrolysis may have been supported by this germ. Our results suggest that the most likely relevant factor in polyurethane degradation is the biological response of MDM and FBGC on outer jacket tubing, which forms the brainelectrode interface. In our previous study, we considered EB as the result of a DBS electrode malfunctioning, whereas here it is shown how the material degradation progresses as the devices remain implanted in vivo, in functioning electrodes too. Overall, EB provokes evident alterations with regard to a lead implanted for a period of 10 years. In a patient with a long-term chronic implantation of a DBS system, such as a young dystonic patient, the outer jacket polymer degradation could deepen and cause a communication between the external biological space and the internal portion of the lead thus exposing the DBS system to a higher risk of malfunctioning, especially in young patients. It is also worth noting that the degenerative process involved only the most superficial part of the outer jacket tubing (Fig. 2). These considerations indicate that the analyzed PU presents good short- and middleterm (10 years) bio-stability in the case of electrodes for DBS purpose. To avoid hardware-related complications, in lifetime implanted electrodes, other materials could be taken into account.

The two classes of elastomers commonly used in implantable medical devices are the polyurethanes and the silicone. In order to minimize their degradation, the modern generation of polyurethanes has been highly optimized by implementing the following properties: higher level of crystallinity, chemical purity, and aliphatic chains, which maximizes the hydrophobicity. Even though an optimized polymer formulation and fabrication could minimize the polymer alteration, hydrolytic degradation cannot be completely eliminated, due to the 
Table 3 Comparison between the chemical composition of the internal insulating jacket of the analyzed electrodes

\begin{tabular}{lrrrrr}
\hline & $\mathrm{C}$ & $\mathrm{F}$ & $\mathrm{O}$ & $\mathrm{N}$ & $\mathrm{Na}$ \\
\hline Patient 1 & $51 \%$ & $45 \%$ & $2 \%$ & $1 \%$ & \\
Patient 1: alterations covering int. ins. jacket & $63 \%$ & $3,5 \%$ & $12 \%$ & $11 \%$ & $7 \%$ \\
Patient 2 & $59 \%$ & $32 \%$ & $5 \%$ & $2 \%$ & $0.5 \%$ \\
Patient 3 & $40 \%$ & $54 \%$ & $3 \%$ & $2 \%$ & $0.5 \%$ \\
Mean (pt 1, 2,3) & $50 \%$ & $43.3 \%$ & & & \\
Control electrode & $38 \%$ & $61 \%$ & $1 \%$ & $0.3 \%$ & \\
Equilibrium & $+12 \%$ & $-17.7 \%$ & & & \\
\hline
\end{tabular}

"in vivo" intrinsic instability of the urethane-group. Silicones, on the other hand, are known to be more stable toward hydrolysis [4]. Nevertheless, corrosion phenomena have been observed also on the silicones surface upon removal [6].

It is also worth mentioning that the described alterations are not directly related to the modality of brain stimulation. DBS parameters are different in dystonic and in epileptic patients: cyclic bipolar stimulation is generally used in the latter cases. In our series, each patient was treated by using continuous stimulation, while dystonic patients were managed through both higher monopolar current intensity (patient 1:3.7 V; patient $3: 3.2$ on the right and 3.9 on the left; versus patient 2: $2 \mathrm{~V}$ ) and frequency (135 Hz and $185 \mathrm{~Hz}$ vs. $100 \mathrm{~Hz}$ ).

Considering the internal insulating jacket, the comparison through SEM between the electrodes removed from patients 2 and 3 (Fig. 4b, c) and the new electrode did not show any alterations [7]. On the contrary, considerable changes were observed in patient 1 (Fig. 4a). In this case, fragments of material with a melted aspect partially covering the internal insulating jacket were found, which was similar to our previous work [7]. These alterations were probably caused by a process that allows the detachment and the molecular rearrangement of the lead material. The analysis of chemical composition by EDX sustains this hypothesis (Tables 2 and 3). In fact, the amount of each chemical element is coherent with the occurrence of a mixture between the polyurethane (forming the outer jacket tubing) and fluoropolymer (forming the internal insulating jacket) (in particular see Table 3: "alterations covering int. ins. Jacket"). In comparison to our previous findings, we can suggest that the degenerated materials found above the internal insulating jacket are probably correlated to the lead impairment, suggesting events, such as overheating with an open-circuit formation [7]. The other analyzed electrodes did not show alterations at this level.

In this preliminary study, neither an accurate statistical analysis nor a quantitative numerical quantification of the damage in SEM analysis was performed. This limitation was mainly due to the small number of cases that we considered.

In conclusion, environmental biodegradation (EB) is a phenomenon that occurs in each long-term implantable medical device. The morphological and chemical characterization of implanted hardware supports the design phase of DBS devices by optimizing the bio-stability of the electrodes and also predicting the risk of impairment. Further data, coming from industrial and pre-clinical studies, are needed to test alternative materials suitable for implantation and to verify the efficacy of new generation of devices [3].

Acknowledgments Daniel R. Bonner for language revision.

\section{References}

1. Benabid AL, Chabardes S, Mitrofanis J, Pollak P (2009) Deep brain stimulation of the subthalamic nucleus for the treatment of Parkinson's disease. Lancet Neurol 8(1):67-81

2. Bucky LP, Ehrlich HP, Sohoni S, May JW Jr (1994) The capsule quality of saline-filled smooth silicone, textured silicone, and polyurethane implants in rabbits: a long-term study. Plast Reconstr Surg 93:1123-1131

3. Corbelli G, Ghisleri C, Marelli M, Milani P, Ravagnan L (2011) Highly deformable nanostructured elastomeric electrodes with improving conductivity upon cyclical stretching. Adv Mater 23(39): $4504-4508$

4. Coutry AJ (1996) Degradation of materials in biological environment. In: Ratner BD, Hoffman AS, Schoen FJ, Lemons JE (eds) Biomaterial science. An introduction to materials in medicine. Elsevier Academic Press, San Diego

5. Franzini A, Cordella R, Messina G, Marras CE, Romito LM, Carella F, Albanese A, Rizzi M, Nardocci N, Zorzi G, Zekay E, Broggi G (2011) Deep brain stimulation for movement disorders. Considerations on 276 consecutive patients. J Neural Transm 118(10):1497-1510

6. Kołodzińska A, Kutarski A, Kozłowska M, Grabowski M, Marchel H, Drela N, Opolski G (2013) Biodegradation of the outer silicone insulation of endocardial leads. Circ Arrhythm Electrophysiol 6(2): 279-286

7. Marras C, Rizzi M, Ravagnan L, De Benedictis A, Zorzi G, Bongiorno G, Marchesi D, Messina G, Cordella R, Franzini A (2013) Morphological and chemical analysis of a deep brain stimulation electrode explanted from a dystonic patient. J Neural Transm 120(10):1425-1431

8. Moss J, Ryder T, Aziz TZ, Graeber MB, Bain PG (2004) Electron microscopy of tissue adherent to explanted electrodes in dystonia and Parkinson's disease. Brain 127(Pt 12):2755-2763

9. Paynter RW, Askill IN, Glick SH, Guidoin R (1988) The hydrolytic stability of mitrathane (a polyurethane urea) an X-ray photoelectron spectroscopy study. J Biomed Mater Res 22:687-698

10. Santerre JP, Duguay DG, Labow RS, Brash JL (1995) Interactions of hydrolytic enzymes at an aqueous-polyurethane interface. Proteins at interfaces II. J Am Chem Soc 602:352-70 
11. Santerre JP, Woodhouse K, Laroche G, Labow RS (2005) Understanding the biodegradation of polyurethanes: from classical implants to tissue engineering materials. Biomaterials 26(35): 7457-7470

12. Sillay KA, Larson PS, Starr PA (2008) Deep brain stimulator hardware-related infections: incidence and management in a large series. Neurosurgery 62(2):360-366 (discussion 366-7)

13. Smith R, Oliver C, Williams DF (1987) The enzymatic degradation of polymers in vitro. J Biomed Mater Res 21:991-1003

14. Wagner H, Beller FK, Pfautsch M (1977) Electron and light microscopy examination of capsules around breast implants. Plast Reconstr Surg 60(1):49-54

15. Weaver FM, Follett K, Stern M, Hur K, Harris C, Marks WJ Jr, Rothlind J, Sagher O, Reda D, Moy CS, Pahwa R, Burchiel K, Hogarth P, Lai EC, Duda JE, Holloway K, Samii A, Horn S, Bronstein J, Stoner G, Heemskerk J, Huang GD, CSP 468 Study Group (2009) Bilateral deep brain stimulation vs best medical therapy for patients with advanced Parkinson disease: a randomized controlled trial. JAMA 301(1):63-73
16. Wiemer M, Butz T, Schmidt W, Schmitz KP, Horstkotte D, Langer C (2010) Scanning electron microscopic analysis of different drug eluting stents after failed implantation: from nearly undamaged to major damaged polymers. Catheter Cardiovasc Interv 75(6):905-911

All authors certify that they have no affiliations with or involvement in any organization or entity with any financial interest (such as honoraria; educational grants; participation in speakers' bureaus; membership, employment, consultancies, stock ownership, or other equity interest; and expert testimony or patent-licensing arrangements), or non-financial interest (such as personal or professional relationships, affiliations, knowledge or beliefs) in the subject matter or materials discussed in this manuscript.

All the patients have consented to the submission of this Experimental Research to the journal Acta Neurochirurgica. 